\title{
The Comparison of Sensitivity and Specificity of ELISA-based Microneutralization Test with Hemagglutination Inhibition Test to Evaluate Neutralizing Antibody against Influenza Virus (H1N1)
}

\author{
Shazaib Akhtar ${ }^{1}$, Bilal Nazar ${ }^{2}$, Andleeb Shariq ${ }^{3}$ \\ 1. House Officer, Services Institute of Medical Sciences, Lahore \\ 2. House Officer, Nishtar Hospital, Multan House Officer \\ 3. Allama Iqbal Memorial teaching Hospital, Sialkot \\ Corresponding Author: dr.andleebshariq@gmail.com
}

\begin{abstract}
:
Objective: To compare the sensitivity and specificity of ELISA-based microneutralization test with hemaglutination inhibition test for the evaluation of neutralizing antibody against influenza virus (H1N1). Study Design: Nonrandomized Quasi Trail. Study Place and Study Duration: Department of Medicine, Allama Iqbal memorial teaching hospital, Sialkot and Nishtar Hospital Multan. January 2018 to May 2019. Material and methods: A total of 188 patients were enrolled in our study out of which 101 suffered from influenza type A. Serum samples were collected from different staff members during this period. ELISA based Microneutralization test was performed in 101 samples and similarly Hemagglutination assay was also performed in 101 serum samples. Tests were run twice on two separate days and final result was average of the two runs. All the data thus obtained was subjected to statistical analysis. Frequency and percentages were calculated for qualitative variables like, gender, sensitivity and specificity, while mean and standard deviation was calculated for quantitative variables like, age and BMI. Results: Both the tests were performed on all the sera samples. The sensitivity of the Hemagglutination Inhibition Test (HI) was $65.34 \%$ and the specificity was $66.51 \%$. On the contrary, the sensitivity was $87.12 \%$ and specificity was $90.80 \%$ on the ELISA based microneutralization assay (micro NT-ELISA). As the sensitivity and specificity of the micro NT-ELISA is more than that of Hemagglutination Inhibition Test, this makes ELISA based microneutralization assay superior.Conclusion: ELISA based microneutralization test is much more sensitive and specific as compared to the hemaglutination inhibitor assay in terms of detection of neutralizing antibodies against influenza virus (H1N1).
\end{abstract}

Keywords: Microneutralization, Hemagglutination, Influenza

DOI: $10.7176 / \mathrm{JMPB} / 65-08$

Publication date: April $30^{\text {th }} 2020$

\section{Introduction:}

Seasonal pandemics and epidemics of influenza result in considerable number of mortalities and morbidities and it is regarded as a major health issue in the entire world ${ }^{1}$. It can be detected with several types of techniques ${ }^{2}$. One of the techniques is serological assays which are very important in terms of detecting the influenza virus ${ }^{3}$. All these techniques are helpful in surveillance, sero-epidemiological studies, development of vaccines and their evaluation and also for diagnosis of influenza especially whenever a new strain of influenza virus is suspected ${ }^{4}$. Particular antibodies against certain viral antigen as humoral immune response, are produced as result of infection by the virus or if vaccination of the virus. These antibodies are detectable for duration of about two to three weeks after the emergence of the symptoms with the help of serological testing ${ }^{5}$. This is helpful in confirming the past infection without the use of complete virological work up. Even in the presence of undetectable virus, a significant rise in antibody titer in serum i.e. more than 4 times the normal, within the duration of acute and convalescent phase is sufficiently enough to make a diagnosis ${ }^{6}$. The advantages of serological assays like, Hemagglutination inhibition test and ELISA based Microneutralization test, are not only that these are diagnostically helpful but also that they provide essential knowledge when use for immunological and epidemiological surveys and also in evaluation of immunogenicity of a vaccine ${ }^{7}$.

Most commonly used serological test for quantification of antibodies against influenza virus antigen is Hemagglutination inhibition test. This test is rendered as gold standard in multiple settings in influenza serological testing and is diagnostic test of choice as well ${ }^{8}$. It is also used for the determination of 
immunogenicity of vaccine and also in seasonal surveys. Two difficulties in performance of this test are that, in HI (Hemagglutination inhibition) test, the results are dependent upon the types of RBCs and non specific inhibitors are inevitably removed from the serum during this test. Other limitations to its use are that HI cannot be used in detection of avian viruses like H5N1 \& H3N2 subtypes. To overcome these limitations of HI, Microneutralization technique has been developed which helps not only in the detection of functional antibodies to influenza viruses and vaccinations and in certain circumstances has proved to be more sensitive as compared to HI test. Microneutralization test is performed in multiple ways including Cytopathic effect formation in cell cultures, RBCs in order to detect released viral antigen or use of ELISA test along with microneutralization in order to detect cells infected with human influenza virus. Studies suggest that ELISA technique is much more time consuming, difficult procedure to perform and is most commonly requires the presence of monoclonal antibodies in the serum which are usually not present in newly infected patients. Another serological test used for the detection of neutralizing antibodies in patients with influenza virus infection is known as colorimetric microneutralization assay.

In ELISA based Microneutralization test, standard serum neutralization test is used while ELISA measures the viral load or number of infected cells in the blood. This can be divided into three consecutive steps; TCID (tissue culture infectious dose) determination, Microneutralization assay of the virus and ELISA. Results can be gathered within two days in this test ${ }^{9}$. Many studies have evaluated the antibody response in people infected with human influenza virus strain $\mathrm{H} 1 \mathrm{~N} 1$ with the help of hemagglutination inhibitor assay. There are also some studies which shown that in terms of sensitivity and specificity in detecting the neutralizing antibodies in serum of the infected people with influenza virus, microneutralization combined with ELISA assay are highly efficient. No such study has been performed previously in this region of the world in order to compare the HI test to Microneutralization-ELISA test for detection of neutralizing antibodies against influenza virus. In this study comparison on the basis of sensitivity and specificity of both tests will be performed so that further recommendation can be made on the basis of the superiority of the either test.

\section{Material and Method:}

This is a simple quasi trail performed in department of medicine in Allama Iqbal memorial teaching hospital, Sialkot and Nishtar Hospital Multan. January 2018 to May 2019. A total of 188 patients were enrolled in our study out of which 101 suffered from influenza type A. Serum samples were collected from different staff members during this period. Ethical approval for this study was obtained by Hospitals Ethics Committee. Sample size was calculated from the reference study by Ahmad Tavakoli et al ${ }^{10}$. Informed consent was obtained from the patients prior to the enrollment into the study. A WHO protocol was followed for performing the methods of both HI assay and Microneutralization-ELISA test. All the staff members with positive polymerase chain reaction or previous vaccination for influenza were included in this study. Exclusion was made in case of acute infection, unwilling patients or if patients had some other fatal co morbidity. In order to remove the non specific haemagglutination inhibitors, receptor destroying enzyme was used to treat all the serum samples. Then samples were further processed by incubating them overnight at $37 \mathrm{C}$ in a water bath. Later on samples were heated at $56 \mathrm{C}$ for thirty minutes to inactivate the receptor destroying enzyme. While performing the microneutralization test incubation was skipped. Following equation was used to calculate the final titer of viral neutralization;

$X=[($ average A490 of virus control wells) - (average A490 of cell control wells) $] / 2+$ (average A490 of CC wells)

Tests were run twice on two separate days and final result was average of the two runs. All the data thus obtained was subjected to statistical analysis. Frequency and percentages were calculated for qualitative variables like, gender, sensitivity and specificity, while mean and standard deviation was calculated for quantitative variables like, age and BMI.

\section{Results:}

Serum sample was obtained from 188 patients, of which 101 patients had recently suffered from influenza. Mean age of all the patients was $32.29 \pm 13.25$ years, and body mass index was $24.90 \pm 2.94 \mathrm{~kg} / \mathrm{m}^{2}$. Table-I

In HI group, positive results were seen in 66 of the diseased persons and 30 of the healthy persons. Negative results were seen in 35 of the diseased persons and 57 of the healthy persons. While in Micro neutralizationELISA group positive result was seen in 88 of the diseased persons and negative results were seen in 13 of the diseased persons. Similarly among the healthy persons in our study 8 showed positive result and 79 showed negative result when microneutralization test was applied to assess the serological response of neutralizing antibodies in patients with influenza A (H1N1. 
Both the tests were performed on all the sera samples. The sensitivity of the Hemagglutination Inhibition Test (HI) was $65.34 \%$ and the specificity was $66.51 \%$. On the contrary, the sensitivity was $87.12 \%$ and specificity was $90.80 \%$ on the ELISA based microneutralization assay (micro NT-ELISA). As the sensitivity and specificity of the micro NT-ELISA is more than that of Hemagglutination Inhibition Test, this makes ELISA based microneutralization assay superior. Table-II

Table-I

\section{Demographic data}

\begin{tabular}{|l|c|}
\hline \multicolumn{1}{|c|}{ Variable } & Group-1 $(\mathbf{n}=\mathbf{1 0 0})$ \\
\hline Age, years $($ mean \pm S.D $)$ & $32.29 \pm 13.25$ \\
\hline Male, $\mathrm{n}(\%)$ & $112(59.57)$ \\
\hline BMI, $\mathrm{kg} / \mathrm{m}^{2}($ mean \pm S.D) & $24.90 \pm 2.94$ \\
\hline
\end{tabular}

Table-II

Positive and Negative Results of HI and micro NT-ELISA

\begin{tabular}{|c|l|c|c|c|}
\hline \multicolumn{2}{|c|}{ TEST } & Diseased & Healthy & Total \\
\hline \multirow{3}{*}{ HI } & Positive & 66 & 30 & 96 \\
\cline { 2 - 5 } & Negative & 35 & 57 & 92 \\
\cline { 2 - 5 } & Total & 101 & 87 & 96 \\
\hline \multirow{2}{*}{ Micro NT-ELISA } & Positive & 88 & 8 & 92 \\
\cline { 2 - 5 } & Negative & 13 & 79 & 188 \\
\cline { 2 - 5 } & Normal & 101 & 87 & 188 \\
\hline
\end{tabular}

Data is mentioned as number.

\section{Discussion:}

In our study, sensitivity and specificity of both tests was compared by performing the test on 101 serum samples gathered from the staff members of the department who were either previously infected or were vaccinated against human influenza virus A. the sensitivity and specificity of hemaglutination inhibitor assay was lower as compared to the sensitivity and specificity of ELISA based microneutralization test used for the detection of neutralizing antibodies against influenza A (H1N1 strain). Specificity of hemaglutination inhibitor assay and ELISA based microneutralization test was $66.51 \%$ and $90.80 \%$ respectively. Similarly sensitivity of HI assay and ELISA based microneutralization test was $65.34 \%$ and $81.12 \%$ respectively. Studies have reported that microneutralization test is very specific and sensitive in terms of detection of antibodies especially strain specific antibodies ${ }^{2}$. Positive neutralization test can be demonstrated as negative infectivity and it illustrates that virus specific antibodies are present in the serum sample.

Cytopathic effect based microneutralization test when compared to ELISA based microneutralization test, ELISA based test has the superiority as it is reported to be less variable than the former assay 11,12 . Hemagglutination assay is considered as gold standard test for serological diagnosis of influenza virus but it has lower sensitivity when detection of antibody against human influenza virus strain H3N2 is required ${ }^{13}$. In this study, a high rate of correlation between the results of the two tests i.e. ELISA based microneutralization test and hemaglutination inhibition assay were comparable and had a high correlation in terms of specificity and sensitivity. In a previous study when a comparison between HI assay and ELISA based microneutralization test was performed results showed that the later one was more sensitive as compared to the HI assay ${ }^{13}$. In previously reported studies hemaglutination inhibitor assay was superior to ELISA based microneutralization test in terms of detecting the antibody titer. HI assay has proved to be superior for detecting higher numbers of antibody titer ${ }^{14}$ but the results of our study show that even though this notion is true about HI assay, the neutralizing property is not significantly reflected by HI assay, therefore making ELISA based neutralization test as highly specific and sensitive for detection of neutralizing antibodies against human influenza virus. Previously multiple studies have been performed to detect antibodies against different influenza virus strains ${ }^{15,16}$ \& 17 .

\section{Conclusion:}

ELISA based microneutralization test is much more sensitive and specific as compared to the hemaglutination inhibitor assay in terms of detection of neutralizing antibodies against influenza virus (H1N1). 


\section{Conflict of interest:}

There was no conflict of interest.

\section{Funding Source:}

No external funding source was used.

\section{References:}

1- Reed C, Biggerstaff M, Finelli L, Koonin LM, Beauvais D, Uzicanin A, Plummer A, Bresee J, Redd SC, Jernigan DB. Novel framework for assessing epidemiologic effects of influenza epidemics and pandemics. Emerg infect dis. 2013;19(1):85.

2- Vemula SV, Zhao J, Liu J, Wang X, Biswas S, Hewlett I. Current approaches for diagnosis of influenza virus infections in humans. Viruses. 2016;8(4):96.

3- Verschoor CP, Singh P, Russell ML, Bowdish DM, Brewer A, Cyr L, Ward BJ, Loeb M. Microneutralization assay titres correlate with protection against seasonal influenza H1N1 and H3N2 in children. PloS one. 2015;10(6):e0131531.

4- Bai T, Zhou J, Shu Y. Serologic study for influenza A (H7N9) among high-risk groups in China. New Engl J Med. 2013;368(24):2339-40.

5- Nath KD, Burel JG, Shankar V, Pritchard AL, Towers M, Looke D, Davies JM, Upham JW. Clinical factors associated with the humoral immune response to influenza vaccination in chronic obstructive pulmonary disease. Inte j chronic obstr. 2014;9:51.

6- Talaat KR, Luke CJ, Khurana S, Manischewitz J, King LR, McMahon BA, Karron RA, Lewis KD, Qin J, Follmann DA, Golding H. A live attenuated influenza A (H5N1) vaccine induces long-term immunity in the absence of a primary antibody response. J infect dis. 2014;209(12):1860-9.

7- He W, Mullarkey CE, Miller MS. Measuring the neutralization potency of influenza A virus hemagglutinin stalk/stem-binding antibodies in polyclonal preparations by microneutralization assay. Methods. 2015;90:95100.

8- Nguyen M, Fries K, Khoury R, Zheng L, Hu B, Hildreth SW, Parkhill R, Warren W. Automated imaging and analysis of the hemagglutination inhibition assay. $\mathrm{J}$ lab automat. 2016;21(2):287-96.

9- Kitikoon P, Vincent AL. Microneutralization assay for swine influenza virus in swine serum. In Animal Influenza Virus 2014 (pp. 325-335). Humana Press, New York, NY.

10- Tavakoli A, Rezaei F, NASAB GS, Adjaminezhad-Fard F, Noroozbabaei Z, Mokhtari-Azad T. The Comparison of Sensitivity and Specificity of ELISA-based Microneutralization Test with Hemagglutination Inhibition Test to Evaluate Neutralizing Antibody against Influenza Virus (H1N1). Iran j public health. 2017;46(12):1690.

11- Stephenson I, Das RG, Wood JM, Katz JM. Comparison of neutralising antibody assays for detection of antibody to influenza A/H3N2 viruses: an international collaborative study. Vaccine. 2007;25(20):4056-63.

12- Stephenson I, Heath A, Major D, Newman RW, Hoschler K, Junzi W, Katz JM, Weir JP, Zambon MC, Wood JM. Reproducibility of serologic assays for influenza virus A (H5N1). Emerg infect dis. 2009;15(8):1250.

13- Rowe T, Abernathy RA, Hu-Primmer J, Thompson WW, Lu X, Lim W, Fukuda K, Cox NJ, Katz JM. Detection of antibody to avian influenza A (H5N1) virus in human serum by using a combination of serologic assays. J clin microbiol. 1999;37(4):937-43.

14- Cheng LW, Huang SW, Huang LM, Chang LY, Shao PL, Kiang D, Wang JR. Comparison of neutralizing and hemagglutination-inhibiting antibody responses for evaluating the seasonal influenza vaccine. $\mathrm{J}$ virol methods. 2012;182(1-2):43-9. 
15- Heckler R, Baillot A, Engelmann H, Neumeier E, Windorfer A. Cross-protection against homologous drift variants of influenza A and B after vaccination with split vaccine. Intervirol. 2007;50(1):58-62.

16- Lavenu A, Valleron AJ, Carrat F. Exploring cross-protection between influenza strains by an epidemiological model. Virus research. 2004;103(1-2):101-5.

17- Skowronski DM, De Serres G, Janjua NZ, Gardy JL, Gilca V, Dionne M, Hamelin ME, Rheaume C, Boivin G. Cross-reactive antibody to swine influenza A (H3N2) subtype virus in children and adults before and after immunisation with 2010/11 trivalent inactivated influenza vaccine in Canada, August to November 2010. Euro surveillance. 2012;17(4):20066. 